\title{
Pancreatectomy With Arterial and Portal Vein Reconstruction for Locally Advanced Pancreatic Cancer - A Case Report and Literature Review
}

\author{
VLADISLAV BRASOVEANU ${ }^{1,2}$, DRAGOS ROMANESCU ${ }^{3}$, ION BARBU ${ }^{1}$, \\ IRINA BALESCU ${ }^{4}$ and NICOLAE BACALBASA ${ }^{5,6,7}$ \\ ${ }^{1}$ Department of Visceral Surgery, Dan Setlacec Center of Gastrointestinal Diseases \\ and Liver Transplantation, Fundeni Clinical Institute, Bucharest, Romania; \\ ${ }^{2}$ Titu Maiorescu University of Medicine and Pharmacy, Bucharest, Romania; \\ ${ }^{3}$ Department of Surgery, Sanador Medical Center, Bucharest, Romania; \\ ${ }^{4}$ Department of Surgery, Ponderas Academic Hospital, Bucharest, Romania; \\ ${ }^{5}$ Carol Davila University of Medicine and Pharmacy, Bucharest, Romania; \\ ${ }^{6}$ Department of Surgery, Center of Excellence in Translational Medicine, \\ Fundeni Clinical Institute, Bucharest, Romania; \\ ${ }^{7}$ Department of Obstetrics and Gynecology, I. Cantacuzino Clinical Hospital, Bucharest, Romania
}

\begin{abstract}
Background/aim: Pancreatic cancer is still associated with poor survival rates due to the fact that it is most often diagnosed at advanced stages of the disease when local invasion is present. However, improvements of surgical techniques have enabled extended resections with curative intent. We present the case of a 43-year-old patient diagnosed with locally invasive pancreatic adenocarcinoma invading the portal vein and the common hepatic artery. Case Report: Surgery with curative intent consisting of pancreatoduodenectomy en bloc with hepatic artery resection and portal vein resection was successfully performed. The right hepatic artery was further anastomosed with the remaining common hepatic artery while the left hepatic artery was reconstructed using a reversed splenic artery patch. The continuity of the portal vein was reestablished by placing a synthetic prosthesis. Conclusion: Combined arterial and venous resections might be useful in order to achieve a good local control of disease in patients with locally advanced pancreatic cancer.
\end{abstract}

This article is freely accessible online.

Correspondence to: Irina Balescu, Ponderas Academic Hospital, Nicolae Caramfil 85a Street, Bucharest, Romania. Tel: +40 724077709, e-mail: irina.balescu@ ponderas-ah.ro

Key Words: Hepatic artery resection, portal vein resection, reconstruction, locally advanced pancreatic cancer.
Pancreatic cancer remains one of the most aggressive malignancies worldwide, associated with poor rates of survival due to the biological aggressiveness of the tumor and to its high chemoresistance $(1,2)$. Moreover, pancreatic tumors tend to remain asymptomatic for a long period of time, being frequently diagnosed at advanced stages of the disease when invasion of vascular structures is already present or when disseminated metastases have developed (3). In such cases, the overall prognosis is extremely poor, with survival of less than 1 year being expected. Therefore, it is estimated that pancreatic cancer currently represents the fourth cause of cancer related death worldwide $(1,2)$. However, the development of vascular techniques of reconstruction have brought a benefit in terms of survival by providing the opportunity for achieving a radical surgical procedure in cases in which there are no distant metastases. As for the types of vascular structures which might provide a benefit in terms of survival if resected, intensive debates have been conducted. Therefore, in cases presenting venous invasion, the most recent guidelines from the International Study Group for Pancreatic Surgery support demonstrate that venous invasion should no longer be considered as a contraindication for resection, similar rates of long-term survival being reported in cases in which venous resection was needed when compared to cases in which standard pancreatic resections were performed (4). However, in cases with arterial invasion, a clear consensus is still lacking, the indication usually being tailored according to the particularities of each case $(2,5,6)$.

In this light, we investigated a 43-year-old male with no significant previous history for diffuse upper abdominal pain 


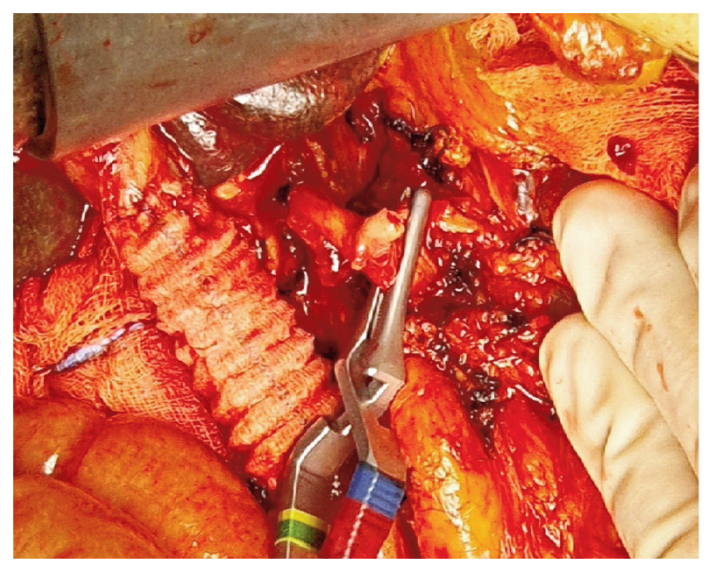

Figure 1. Intraoperative aspect after resection and portal vein reconstruction using a synthetic prosthesis. The celiac trunk is clamped.

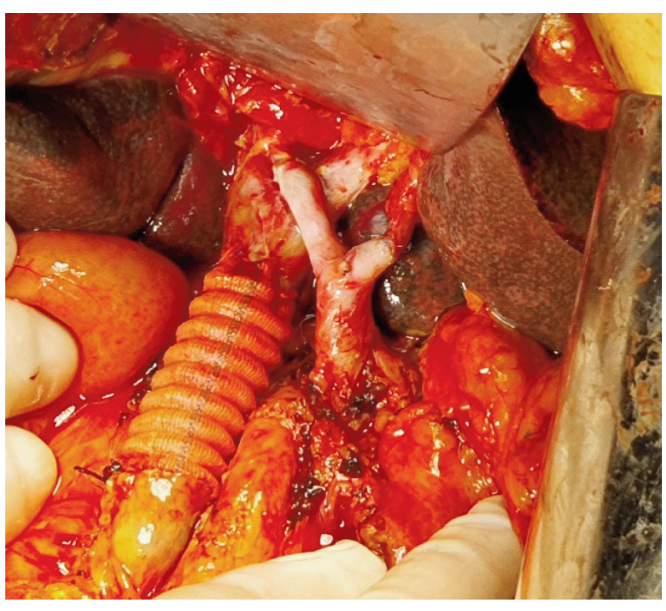

Figure 2. The final aspect after arterial and venous reconstruction.

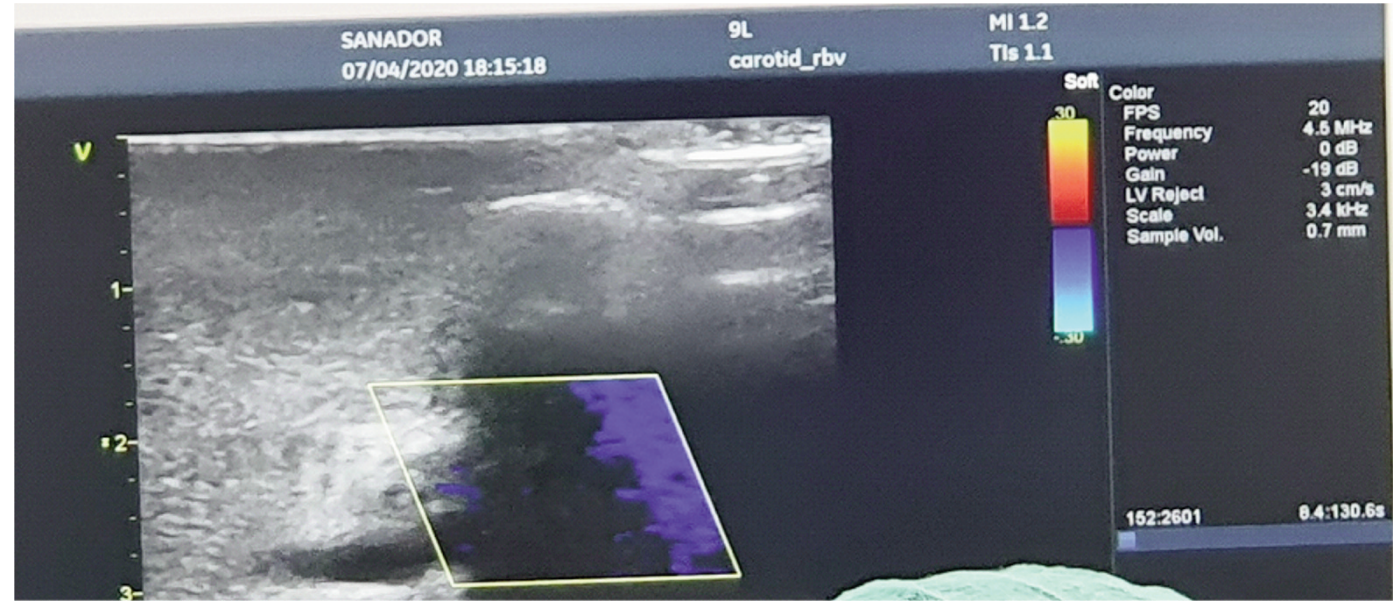

Figure 3. Intraoperative ultrasound demonstrating the presence of adequate hepatic blood flow.

and weight loss who was diagnosed with a locally invasive tumor of the pancreatic head.

\section{Case Report}

The biopsy retrieved by endoscopic ultrasound revealed the presence of a moderately differentiated pancreatic adenocarcinoma; the method demonstrated the presence of portal vein invasion. The imaging studies were completed by performing computed tomography, which confirmed the presence of a tumor of the pancreatic head measuring $4.2 \times 3.5 \times 3.8 \mathrm{~cm}$, with portal vein and hepatic artery invasion. Therefore, the patient was submitted to

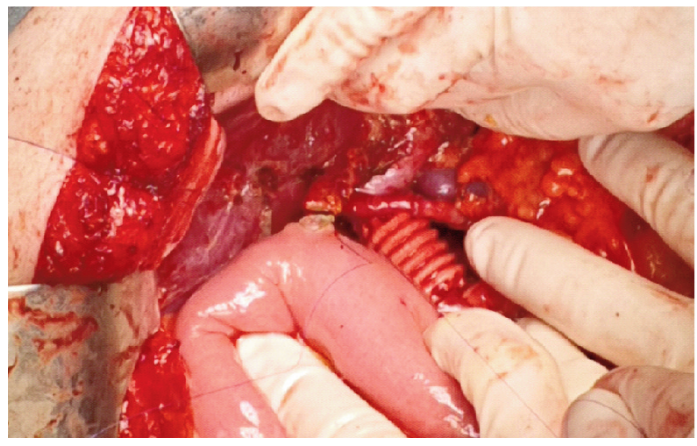

Figure 4. Bilio-enteric anastomosis. 


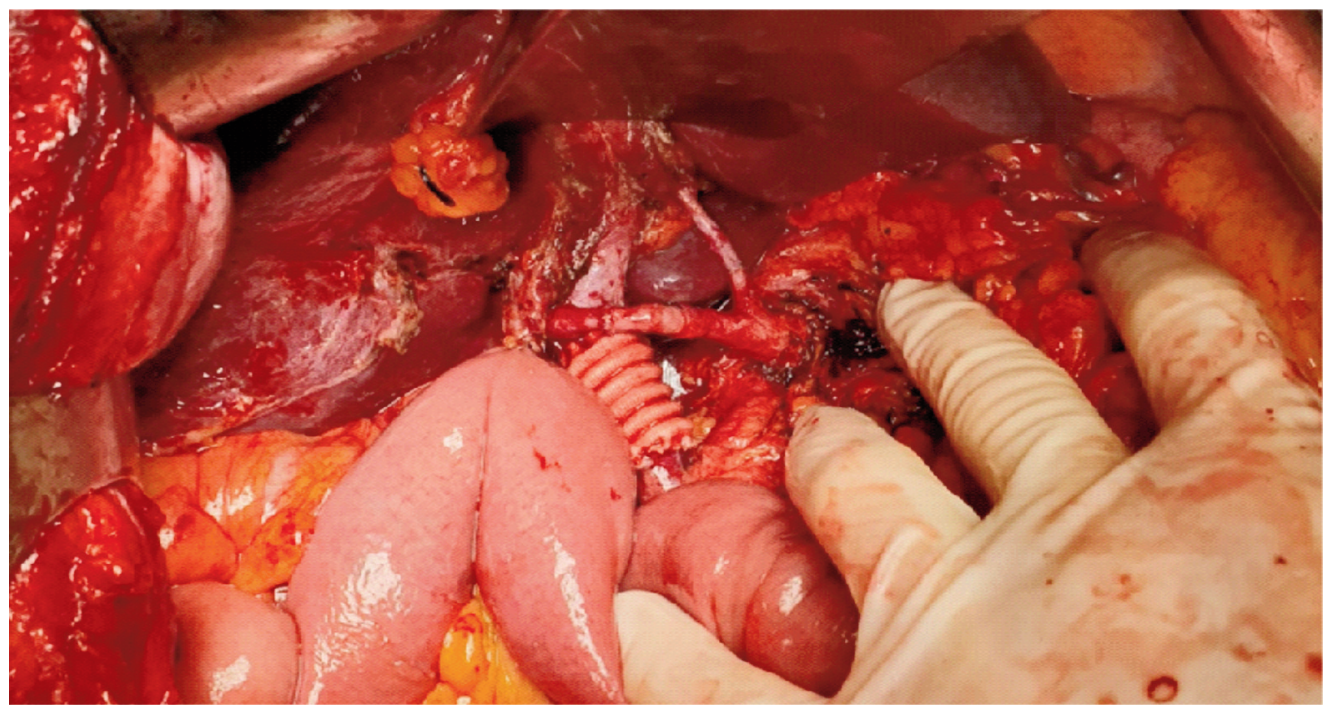

Figure 5. The final aspect after vascular and biliary reconstruction.

irinotecan, oxaliplatin, 5-fluorouracil/leucovorin FOLFIRINOX-based neoadjuvant chemotherapy. One month after completing neoadjuvant chemotherapy another imagistic evaluation was performed, demonstrating a global decrease of the pancreatic head tumor (which now measured $3.9 \times 3.5 \times 3 \mathrm{~cm}$ ), as well as an apparently disappearance of the hepatic artery invasion; however, the portal vein was still infiltrated. The patient was submitted to surgery with curative intent, the preoperative intention being a pancreatoduodenectomy en bloc with portal vein resection and reconstruction. Intraoperatively, persistence of the common and proper hepatic artery was found in association with portal vein infiltration; however, tumoral infiltration of the arterial structures proved to be on less than $180^{\circ}$ of the vascular circumference. Consequently, the decision was made to continue and to perform surgery with curative intent; pancreatoduodenectomy en bloc with portal vein resection, common and proper hepatic artery resection were performed. The right hepatic artery was anastomosed with the remaining common hepatic artery, while the left hepatic artery was anastomosed with a reversed patch from the splenic artery by means of micro-surgery. The portal vein was reconstructed using a synthetic prosthesis (Figures 1-5). Doppler ultrasound confirmed the presence of adequate blood flow at the hepatic level, while the biochemical tests at 48 hours postoperatively demonstrated a slight increase of the serum levels of liver enzymes (aspartate aminotransferase levels measuring 133 $\mathrm{U} / 1$ ). The patient was discharged with a satisfactory general status on the seventh postoperative day.

\section{Discussion}

Due to the close proximity of the pancreatic head with major vascular structures, in a significant number of cases venous and even arterial invasion might be encountered in cancer of the pancreatic head. Such cases have been considered for a long time as unresectable and were submitted to palliative chemotherapy; however, the long-term outcomes remained very poor. For this reason, since techniques of vascular surgery improved, vascular resections have been successfully incorporated into the therapeutic armamentarium for cancer of the pancreatic head (6-12).

However, while the utility of venous resection has been widely demonstrated, similar rates of survival being reported for standard pancreatoduodenectomy and pancreatoduodenectomy and venous resections, the issue of arterial resection and reconstruction has been widely debated $(2,4$, $6,7)$.

Although the rates of long-term survival after pancreatic resection en bloc with arterial resection and reconstruction seem to be poorer when compared to cases submitted to venous resection, these rates seem to be better when compared to cases submitted to palliative treatment $(2,6-8)$. A study conducted on this issue was published in 2019 by Del Chiaro et al. (2); it included 73 patients submitted to resection for borderline or locally advanced pancreatic cancer, their outcomes being compared to those of patients diagnosed at similar stages of the disease but submitted only to palliative surgery and chemotherapy. The authors demonstrated that although the rates of postoperative complications were similar for the two groups, the 1-, 3- and 
5-year overall survival rates were significantly higher among cases submitted to surgery with curative intent. Regarding the types of vascular reconstructions, all cases necessitating portal vein resections benefited from end-to-end anastomosis, while in cases in which arterial resection was needed, reconstruction consisted of end-to-end anastomosis in $47 \%$ of cases, followed by the rotation of the splenic artery in $31 \%$ of cases. Other arterial reconstructions consisted of anastomosis on the gastroduodenal stump, autologous or synthetic graft interposition or Appleby procedure; however, the proportion of these reconstructions was significantly lower when compared to direct end-to-end anastomosis or splenic replacement. Interestingly, the authors underlined that the association of neoadjuvant chemotherapy did not significantly influence the long-term outcomes in cases in which radical surgery was achievable. Moreover, there was no significant difference in terms of survival between patients with and without histologically proven arterial infiltration, while cases in which isolated arterial resection was needed tended to have a more favorable outcome when compared to those in which both arterial and venous resections were needed. Therefore, the authors concluded that there is hope for arterial resection to have a similar fate to venous resection in locally advanced pancreatic cancer. While initially it was considered that the presence of venous invasion should be considered as a formal contraindication for resection in pancreatic cancer, further studies have demonstrated that once the perioperative management of these patients has improved, similar longterm outcomes are to be expected in cases submitted to standard resection or to pancreatoduodenectomy in association with venous resections $(3,8,13,14)$. In this respect, Del Chiaro et al. concluded that arterial resection may become widely accepted and part of the standard therapeutic protocol for locally advanced pancreatic cancer with arterial involvement (2).

A similar conclusion was also presented by Amano et al. after studying the long-term outcomes of 17 patients submitted to neoadjuvant chemotherapy followed by pancreatoduodenectomy en-bloc with arterial resection in an article published in 2015 (15). However, in that study, neoadjuvant therapy consisted of gemcitabine and neoadjuvant irradiation, while in Del Chiaro et al.'s study (2), most patients were submitted to the FOLFIRINOX protocol of neoadjuvant chemotherapy.

In regard to the prognostic factors after extended resection for locally advanced pancreatic cancer after neoadjuvant treatment, a recent study conducted by Maeda et al. demonstrated that the presence of arterial invasion did not significantly influence the long-term outcomes as long as negative resection margins were achieved (16); in their study, the authors included 305 patients from three tertiary centers diagnosed with borderline resectable pancreatic cancer submitted to neoadjuvant chemotherapy or chemo-irradiation followed by surgery. They reported a median overall survival of 29.8 months; elderly patients, cases with positive resection margins, presenting poorly differentiated tumors, positive lymph nodes, or positive perineural invasion, as well as those who required administration of adjuvant chemotherapy, had significantly poorer long-term survival rates. Among these 305 cases, 17 required arterial resection followed by reconstruction, negative resection margins being encountered in $88.2 \%$ of cases; however, the need for performing arterial resection itself was not a significant prognostic factor for poorer long-term outcomes as long as negative resection margins were achieved (16).

\section{Conclusion}

Combined arterial and venous resections seem to be feasible and effective in order to achieve curative resection in locally advanced pancreatic cancer. However, it should be stressed that this surgical procedure is a very demanding one, an attentive selection of cases being mandatory. Although arterial resection is currently not part of the standard therapeutic protocol for locally advanced pancreatic cancer, most recent studies report promising results, which enable us to consider that in the next decade, arterial resection will be considered along with venous resection as feasible and safe procedures in cases diagnosed with locally advanced lesions.

\section{Conflicts of Interest}

The Authors have no conflicts of interest to declare regarding this study.

\section{Authors' Contributions}

V. Brasoveanu, D. Romanescu and I. Barbu performed the surgical procedure; I. Balescu reviewed literature data and prepared the draft of the article; N. Bacalbasa reviewed the final version of the article. All Authors read and approved the final version of the article.

\section{References}

1 Rahib L, Smith BD, Aizenberg R, Rosenzweig AB, Fleshman JM and Matrisian LM: Projecting cancer incidence and deaths to 2030: the unexpected burden of thyroid, liver, and pancreas cancers in the United States. Cancer Res 74(11): 2913-2921, 2014. PMID: 24840647. DOI: 10.1158/0008-5472.CAN-14-0155

2 Del Chiaro M, Rangelova E, Halimi A, Ateeb Z, Scandavini C, Valente R, Segersvard R, Arnelo $U$ and Verbeke CS: Pancreatectomy with arterial resection is superior to palliation in patients with borderline resectable or locally advanced pancreatic cancer. HPB 21(2): 219-225, 2019. PMID: 30093144. DOI: $10.1016 /$ j.hpb.2018.07.017

3 Boggi U, Del Chiaro M, Croce C, Vistoli F, Signori S, Moretto C, Amorese G, Mazzeo S, Cappelli C, Campani D and Mosca F: 
Prognostic implications of tumor invasion or adhesion to peripancreatic vessels in resected pancreatic cancer. Surgery 146(5): 869-881, 2009. PMID: 19744432. DOI: 10.1016/j.surg.2009.04.029

4 Bockhorn M, Uzunoglu FG, Adham M, Imrie C, Milicevic M, Sandberg AA, Asbun HJ, Bassi C, Buchler M, Charnley RM, Conlon K, Cruz LF, Dervenis C, Fingerhutt A, Friess H, Gouma DJ, Hartwig W, Lillemoe KD, Montorsi M, Neoptolemos JP, Shrikhande SV, Takaori K, Traverso W, Vashist YK, Vollmer C, Yeo CJ and Izbicki JR: Borderline resectable pancreatic cancer: A consensus statement by the International Study Group of Pancreatic Surgery (ISGPS). Surgery 155(6): 977-988, 2014. PMID: 24856119. DOI: 10.1016/j.surg.2014.02.001

5 Blazer M, Wu C, Goldberg RM, Phillips G, Schmidt C, Muscarella P, Wuthrick E, Williams TM, Reardon J, Ellison EC, Bloomston $\mathrm{M}$ and Bekaii-Saab T: Neoadjuvant modified (m) FOLFIRINOX for locally advanced unresectable (LAPC) and borderline resectable (BRPC) adenocarcinoma of the pancreas. Ann Surg Oncol 22(4): 1153-1159, 2015. PMID: 25358667. DOI: $10.1245 / \mathrm{s} 10434-014-4225-1$

6 Hackert T, Sachsenmaier M, Hinz U, Schneider L, Michalski CW, Springfeld C, Strobel O, Jager D, Ulrich A and Buchler MW: Locally advanced pancreatic cancer: neoadjuvant therapy with FOLFIRINOX results in resectability in $60 \%$ of the patients. Ann Surg 264(3): 457-463, 2016. PMID: 27355262. DOI: 10.1097/SLA.0000000000001850

7 Brasoveanu V, Anghel C, Barbu I, Pautov M, Ionescu MI, Motthor M, Balescu I, Dima $\mathrm{S}$ and Bacalbasa N: Pancreatoduodenectomy en bloc with portal and superior mesenteric artery resection - a case report and literature review. Anticancer Res 35(3): 1613-1618, 2015. PMID: 25750318.

8 Brasoveanu V, Dumitrascu T, Bacalbasa N and Zamfir R: Splenic artery used for replaced common hepatic artery reconstruction during pancreatoduodenectomy - a case report. Chirurgia 104(4): 499-504, 2009. PMID: 19886062.

9 Bacalbasa N, Brezean I, Anghel C, Barbu I, Pautov M, Balescu I and Brasoveanu V: Successful resection and vascular ligation of a large hepatic artery aneurysm - a case report and literature review. In Vivo 31(5): 979-982, 2017. PMID: 28882969. DOI: 10.21873/invivo. 11157

10 Bacalbasa N, Brezean I, Anghel C, Barbu I, Pautov M, Balescu $\mathrm{I}$ and Brasoveanu V: Management of a fulminant upper gastrointestinal bleeding exteriorized through hemobilia due to arteriobiliary fistula between the common bile duct and a right hepatic artery aneurysm - a case report. In Vivo 31(5): 983-989, 2017. PMID: 28882970. DOI: 10.21873/invivo.11158
11 Bacalbasa N, Balescu I, Tanase A, Pautov M, Brezean I, Vilcu $\mathrm{M}$ and Brasoveanu V: Spleno-pancreatectomy en bloc with parcelar gastrectomy for splenic artery aneurysm - a case report and literature review. In Vivo 32(4): 915-919, 2018. PMID: 29936480. DOI: 10.21873/invivo.11329

12 Bacalbasa N, Balescu I, Tanase A, Brezean I, Vilcu M and Brasoveanu V: Successful resection of a non-functional paraganglioma with celiac trunk invasion followed by common hepatic artery reimplantation - a case report and literature review. In Vivo 32(4): 911-914, 2018. PMID: 29936479. DOI: 10.21873/invivo. 11328

13 Petrelli F, Coinu A, Borgonovo K, Cabiddu M, Ghilardi M, Lonati V, Aitini E and Barni S: FOLFIRINOX-based neoadjuvant therapy in borderline resectable or unresectable pancreatic cancer: A metaanalytical review of published studies. Pancreas 44(4): 515-521, 2015. PMID: 25872127. DOI: 10.1097/MPA.0000000000000314

14 Torgeson A, Lloyd S, Boothe D, Tao R, Whisenant J, GarridoLaguna I and Cannon GM: Multiagent induction chemotherapy followed by chemoradiation is associated with improved survival in locally advanced pancreatic cancer. Cancer 123(19): 38163824, 2017. PMID: 28621885. DOI: 10.1002/cncr.30780

15 Amano R, Kimura K, Nakata B, Yamazoe S, Motomura H, Yamamoto A, Tanaka S and Hirakawa K: Pancreatectomy with major arterial resection after neoadjuvant chemoradiotherapy gemcitabine and S-1 and concurrent radiotherapy for locally advanced unresectable pancreatic cancer. Surgery 158(1): 191200, 2015. PMID: 25900035. DOI: 10.1016/j.surg.2015.02.016

16 Maeda S, Moore AM, Yohanathan L, Hata T, Truty MJ, Smoot RL, Cleary SP, Nagorney DM, Grotz TE, Park EJ, Girgis MD, Reber HA, Motoi F, Masuda T, Unno M, Kendrick ML and Donahue TR: Impact of resection margin status on survival in pancreatic cancer patients after neoadjuvant treatment and pancreatoduodenectomy. Surgery 167(5): 803-811, 2020. PMID: 31992444. DOI: 10.1016/j.surg.2019.12.008

Received April 23, 2020

Revised May 10, 2020

Accepted May 15, 2020 